\title{
Completely Stopped and Dispersionless Light in Plasmonic Waveguides
}

\author{
Kosmas L. Tsakmakidis," Tim W. Pickering, Joachim M. Hamm, A. Freddie Page, and Ortwin Hess ${ }^{\dagger}$ \\ Blackett Laboratory, Department of Physics, Imperial College London, London SW7 2AZ, United Kingdom
}

(Received 2 April 2013; published 25 April 2014)

\begin{abstract}
We introduce a scheme where a time-dependent source excites "complex-frequency" modes in uniform plasmonic heterostructures, enabling complete and dispersionless stopping of light pulses, resilient to realistic levels of dissipative, radiative, and surface-roughness losses. Using transparent conducting oxides at telecommunication wavelengths we show how, without increasing optical losses, multiple light pulses can decay with time precisely at their injection points, unable to propagate despite the complete absence of barriers in front or behind them. Our results theoretically demonstrate extraordinary large light-deceleration factors (of the order of $1.5 \times 10^{7}$ ) in integrated nanophotonic media, comparable only to those attainable with ultracold atomic vapors or with quantum coherence effects, such as coherent population oscillations, in ruby crystals.
\end{abstract}

DOI: 10.1103/PhysRevLett.112.167401

PACS numbers: 78.67.Pt, 42.25.Bs, 78.20.Ci, 78.45.+h

The extremely large speed of light is a tremendous asset but also makes it challenging to control, store, or shrink beyond its wavelength. Particularly, reducing the speed of light [1] down to zero is of fundamental scientific interest $[2,3]$ that could usher in a host of important photonic applications, some of which are as yet fundamentally inaccessible. These include cavity-free, low-threshold nanolasers [4-7], novel solar cell designs for efficient harvesting of light $[8,9]$, nanoscale quantum information processing (owing to the enhanced density of states) $[7,10]$, as well as enhanced biomolecular sensing $[6,11]$.

Until now, complete stopping of light pulses-leading to their localization in given regions of space, in solid integrated structures and at ambient conditions-has been hampered by fundamental difficulties. Ultraslow light requires strong group-index $\left(n_{g}\right)$ resonances, but the increased damping (compared with gases) at normal conditions invariably broadens and weakens such resonances [3]. Certain resourceful schemes, such as electromagnetically induced transparency (EIT), can exploit very narrow absorption dips arising from quantum interference effects to drastically slow down light in ultracold atoms (where damping is minimized), [12] but their solid-state implementations usually decelerate light by up to a factor of a few hundreds $[13,14]$. Furthermore, at the zero group-velocity (zero- $v_{g}$ ) point of an EIT scheme, a light pulse relinquishes its photonic character by coherently mapping its optical quantum states to stationary electronic (spin) excitations [3,15]. Light is "stored" in this way [16], but the ultimate goal of observing and harnessing photons at a zero- $v_{g}$ point is not completely satisfied-even under extreme conditions entailing vacuum operation and ultralow temperatures. Periodic photonic structures offer another route for slowing down light, essentially via periodic backreflections by a lattice of scatterers having sizes comparable to the wavelength [17]. Here, it is structural disorder that fundamentally limits the attainment of light stopping. Tiny nanometer-scale imperfections destroy the (theoretically assumed) "perfect" periodicity, leading to a "smearing out" effect in the attained group indices at the band edges [18]. Practically, this results in slowing down factors that normally do not exceed a few hundreds [19].

We here report on a solid-state configuration leveraging media with negative electromagnetic parameters $[3,20]$, whereby an arbitrary number of light pulses can be stopped, remaining stationary and broadening free at predefined spatial locations, despite the absence of barriers in front of or behind the pulses.

Consider the plasmonic heterostructure shown in Fig. 1(a). The structure supports longitudinally $(x)$ guided, bound, and leaky eigenmodes. We have calculated the structure of the complex dispersion relations $\omega(\beta)$ ( $\beta$ being the longitudinal propagation constant) using the transfer matrix and the argument principle methodology (see the Supplemental Material [21]). The result of our calculations for the $\mathrm{TM}_{2}$ complex modes is illustrated in Fig. 1(b). For modes characterized by real frequency $(\omega)$ and complex wave vector $(\beta)$ we see that, similar to dielectric or atomic configurations, the presence of a decoherence mechanism destroys the singular zero- $v_{g}$ point. However, here, the same configuration also supports another class of modes that, as it turns out, fully uphold the light-stopping condition despite the presence of dissipative losses [see Fig. 1(b)]. These modes, which are frequency poles of the complex Green's function, belong to the complexfrequency (or complex-time, $t$ ), real-wave vector domain $[25,26]$, but it is not immediately clear how or whether they can be accessed, separated from a possible simultaneous excitation of complex- $\beta$ modes (that do not stop), and "cleanly" observed. 
(a)

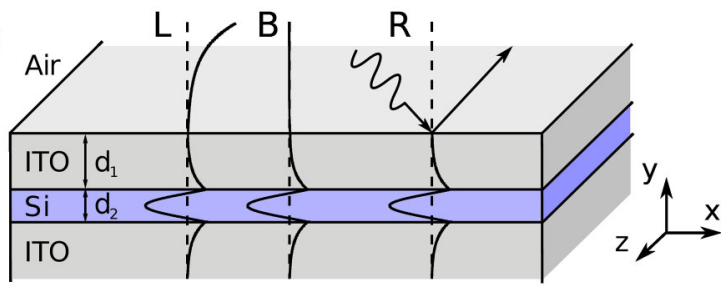

(b)

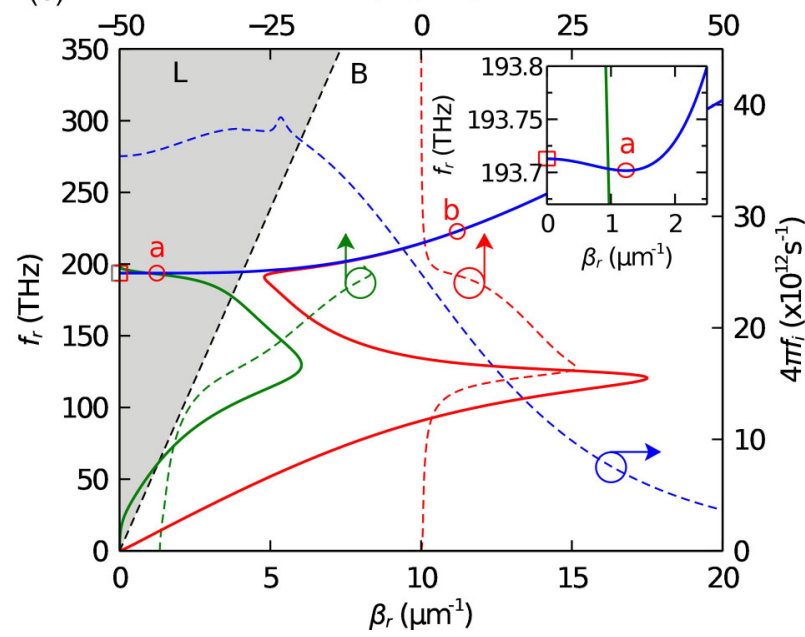

FIG. 1 (color online). (a) Schematic illustration of the $\mathrm{TM}_{2}$ leaky (L), bound (B), and radiation (R) modes of the deployed plasmonic heterostructure. (b) Dispersion diagram of the $\mathrm{TM}_{2}$ complex- $\omega$ (blue) and complex- $k$ (red and green) modes of the ITO/Si/ITO plasmonic heterostructure. Solid lines show the real parts of the frequency $\left[f_{r}=\operatorname{Re}\{\omega\} /(2 \pi)\right]$ and the longitudinal propagation constant $\left(\beta_{r}=\operatorname{Re}\{\beta\}\right)$, while the dashed lines show the corresponding imaginary parts $\left(f_{i}=\operatorname{Im}\{\omega\} /(2 \pi), \quad \beta_{i}=\right.$ $\operatorname{Im}\{\beta\}$ ). The complex- $\omega$ mode is associated with temporal losses, and the two complex- $k$ modes with spatial losses. Note how only the complex- $\omega$ state retains the zero- $v_{g}$ point at point a. For the permittivity of ITO we use the experimental Drude parameters of [27], i.e., $\epsilon_{\infty}=4, \omega_{p}=3.13 \times 10^{15} \mathrm{rad} / \mathrm{s}, \Gamma=1.07 \times 10^{14} \mathrm{~s}^{-1}$, while for $\mathrm{Si}$ we have $\varepsilon_{\mathrm{Si}}=11.68$. Note that the zero- $v_{q}$ point a of the complex- $\omega$ state is on the left-hand side of the air light line (black solid), indicating that the mode is therein weakly leaky. A further zero- $v_{q}$ point occurs at $\beta=0$, making the complex- $\omega$ band very flat in the region around a.

We have found a configuration, illustrated schematically in Fig. 2(a), that manages to simultaneously fulfill the above requirements. We use a silicon slab of thickness $290 \mathrm{~nm}$, bounded by a low-loss plasmonic material (indium tin oxide, ITO) [27]. The upper ITO cladding layer, through which the injection of the light pulses into the middle Si layer is performed, has a finite thickness of $500 \mathrm{~nm}$. At near-infrared wavelengths $(1.55 \mu \mathrm{m})$ and for $p$-polarized light, the (real part of the) permittivity $\varepsilon$ of the ITO layers is negative, thereby providing the required light-deceleration and stopping mechanism $[28,29]$.

More specifically, in our time-domain excitation where the boundary conditions at the excitation plane do not fix

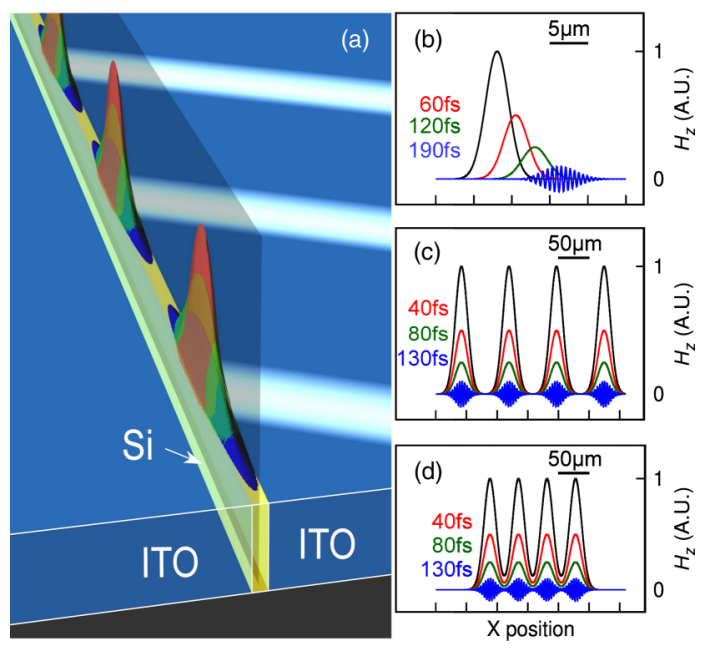

FIG. 2 (color online). (a) Schematic illustration of the configuration used for obtaining complex- $\omega$ stopping of light. The structure, operating at telecommunication wavelengths, is made of a Si slab bounded by two ITO layers, with the upper one (right) having a finite thickness (here not finite for illustrative purposes). The excited complex- $\omega$ mode is weakly leaky at its zero- $v_{g}$ point; hence, there is no need to use a prism. The incident light beams (white), being side by side, directly excite the complex- $\omega$ mode at its zero- $v_{g}$ point at different spatial locations along the waveguide. Rather than being guided along the structure, the excited pulses (in colors) remain stopped (decaying with time at their injection points) without diffusing and broadening at late times. (b)-(d) Full-wave finite-difference time-domain (FDTD) calculations of the time evolution of light pulses injected inside the light-stopping configuration of (a). In all cases the results are recorded on the vertical plane (translucent) cutting through the middle layer. (b) When point b of the dispersion diagram of Fig. 1(b) is hit (where the group velocity is nonzero), a pulse incoupled into the plasmonic structure moves away from its initial point, both decaying and broadening with time. (c) Shown here are four pulses incoupled into the plasmonic heterostructure at its zero- $v_{g}$ point [see also Fig. 2(a)]. The pulses remain stopped, without broadening, for more than $130 \mathrm{fs}$. (d) The pulses in (c) can also be brought closer together - up to the diffraction limit-since the structure is completely uniform, and the localization is not aided by disorder or a cavitylike action. In both (c) and (d) the results have been recorded for times $t>1.05$ ps [cf. Fig. 3(a)].

the frequency to a real value, the evanescent incoupling of a light pulse into the lossy plasmonic slab conserves the reality of the wave vector, allowing for the selective excitation of complex- $\omega$ states at the (singular) zero- $v_{g}$ point. We expect that in the $\beta=0$ (quasistatic) region the bands of the supported modes become flat $\left(v_{g}=0\right)$ [30]; therefore, to minimize group-velocity dispersion and to make the desired band as flat as possible at a finite- $\beta$ zero$v_{g}$ point, such a point must be brought as close as possible to the $\beta=0$ region. Figure 1(b) shows that the design of the above ITO/Si/ITO heterostructure indeed accomplishes this goal, supporting a bulk complex- $\omega$ mode with a zero- $v_{g}$ point on the left-hand side of the air light line. In addition 
to low group-velocity dispersion and preservation of the light-stopping point (in the presence of losses), this weakly leaky mode features two further favorable characteristics. First, since $\beta$ was designed to be finite but small at the zero- $v_{g}$ point, the effective index $n_{\text {eff }}=\beta / k_{0}$ of the mode therein turns out to be smaller than unity: $n_{\text {eff }}=0.3<1$. Consequently, the wavelength $\lambda_{\text {eff }}$ inside the structure becomes larger than the free space one $\left(\lambda_{0}=1.55 \mu \mathrm{m}\right)$, which further diminishes the effect of nanometer-scale surface roughness. Second, since the mode at the zero- $v_{g}$ point is (weakly) leaky, there is no need to utilize a prism to excite it from air, thereby considerably alleviating the realization of the scheme.

To further illustrate these points, Figs. 2(c)-(d) illustrate the time evolution of four light pulses evanescently coupled into the above plasmonic heterostructure by four corresponding exciting beams. The incident free-space wavelength was $\lambda_{0}=1.55 \mu \mathrm{m}(\omega \approx 1.2 \mathrm{PHz})$ and the angle of incidence was $\theta=17.6^{\circ}$, both selected such that precisely the zero- $v_{g}$ point a in Fig. 1(b) was hit. We see from Fig. 2(c) that the four light pulses are completely stopped; they decay with time, without propagating and broadening. From Fig. 1(b) the $e^{-1}$ lifetime of each pulse is calculated to be $28.4 \mathrm{fs}$, while the time taken until the pulses are completely absorbed is approximately $131 \mathrm{fs,} \mathrm{in} \mathrm{agreement}$ with the FDTD calculations of Fig. 2(c). By contrast, when the angle of incidence of an exciting pulse is such that point $\mathrm{b}$ in Fig. 1(b) is hit (where $v_{g} \neq 0$ ), the pulse injected into the heterostructure propagates away from its starting point, decaying but also dispersing (broadening) with time [Fig. 2(b)], as expected. Importantly, while the deceleration factor $\left(n_{g}\right)$ from point $\mathrm{b}\left(n_{g}^{b} \approx 6.6\right)$ to point a $\left(n_{g}^{a} \rightarrow \infty\right)$ increases dramatically, the temporal losses increase by no more than a factor of 2 (from $2.4 \times 10^{13} \mathrm{~s}^{-1}$ to $3.6 \times 10^{13} \mathrm{~s}^{-1}$ ). This crucial aspect, together with the fact that the lifetimes at the stopping point ( $28.4 \mathrm{fs}$ and $131 \mathrm{fs}$ ) are typical nanoplasmonic ones [31], suggests that a wealth of applications within this field should also be possible in the stopped-light regime. Note, further, from Fig. 2(d) that since the herein observed localization is not a cavityor disorder-aided localization, the optical pulses can be injected and stopped at any point along the structure, as well as brought tighter together - up to the diffraction limit. In Fig. 2(d) the four exciting beams (not shown) were brought closer together, so that the four generated pulses inside the slab were also correspondingly tighter together.

The dynamics of the light-stopping mechanism are illustrated in Fig. 3(a), showing an exemplary plot of $\langle x\rangle$ and $\sigma$ (see the Supplemental Material, Sec. 4 [21]) for a single pulse injected close to the zero- $v_{g}$ point. We see that when the source (blue line) is switched off at $t=0.8 \mathrm{ps}$, it causes both $\langle x\rangle$ and $\sigma$ to undergo a transitory behavior (for $0.8 \mathrm{ps}<t<1.05 \mathrm{ps}$ ), until they find their new stable values. In the region $(t>1.05 \mathrm{ps})$ where the immobilized pulse decays freely at its slightly new position, it can be
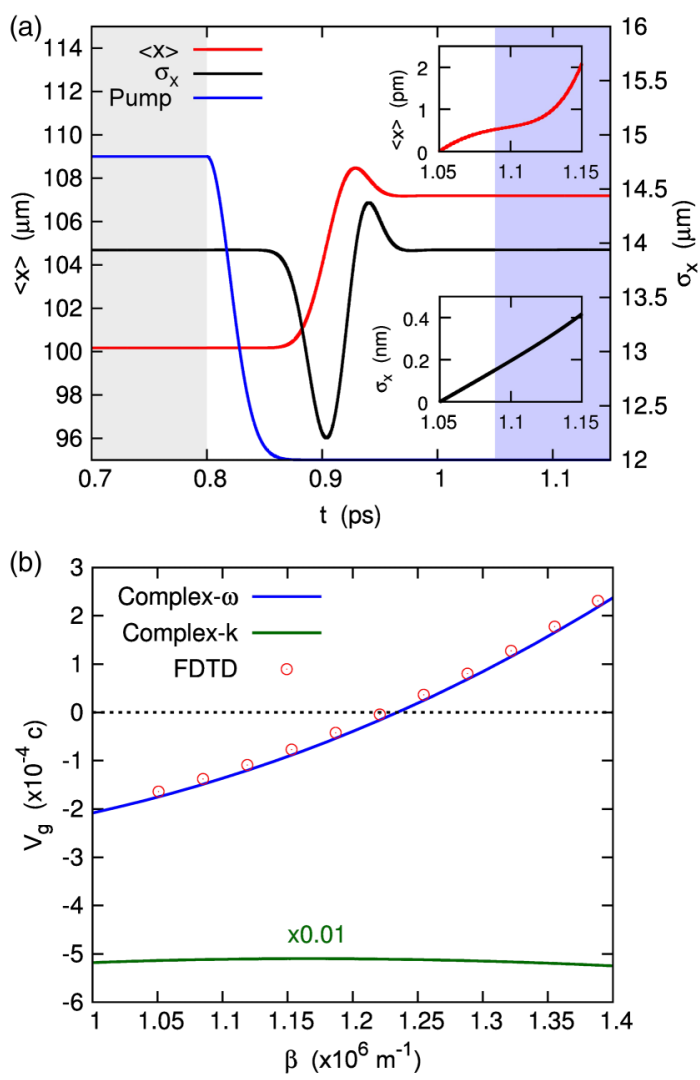

FIG. 3 (color online). (a) FDTD calculations of the variations with time of the mean energy position $\langle x\rangle$ of the excited pulse inside the waveguide (red). Also shown is the temporal variation of the pulse's width $\sigma_{x}$ (black) and the source time signal (blue). Note how, after a transient interval $(0.8-1.05 \mathrm{ps})$, the pulse becomes stopped (red line for $t>1.05 \mathrm{ps),} \mathrm{without} \mathrm{broadening}$ with time (black line for $t>1.05 \mathrm{ps).} \mathrm{The} \mathrm{two} \mathrm{insets} \mathrm{show,} \mathrm{in}$ more detail, the temporal variations of the two curves (for $t>1.05 \mathrm{ps}$ ) from their equilibrium values at $t=1.05 \mathrm{ps}$. (b) Comparison between analytically (lines) and FDTD (symbols) calculated group velocity, $v_{g}$, of the $\mathrm{TM}_{2}$ complex- $\omega$ and complex- $k$ modes, showing unambiguously that in the time domain it is the complex- $\omega$ mode that is excited and attains zero group velocity.

seen (inset) that the center-of-energy position propagates just $2 \mathrm{pm}$ in $100 \mathrm{fs}$ (a velocity of $\sim 20 \mathrm{~ms}^{-1}$ ) proving that incredibly low velocities and ultralarge group indices $\left(n_{g}\right.$ of the order of $1.5 \times 10^{7}$ ) are attainable even in the presence of realistic metallic losses. Further, we find that the pulse only broadens by $0.4 \mathrm{~nm}$; i.e., it is not only immobilized but also does not diffuse or broaden with time. In Fig. 3(b) we present a comparison between the FDTD-calculated center-of-energy velocity [21,32] with both the analytically calculated complex- $\omega$ - and complex- $k$-mode group velocities. We find an excellent agreement between the FDTD and the complex- $\omega$ calculations, showing clearly that in the time domain it is the complex- $\omega$ rather than the customary complex- $k$ description that correctly captures the evolution of ultraslow and stopped light pulses in lossy systems. We 
(a)

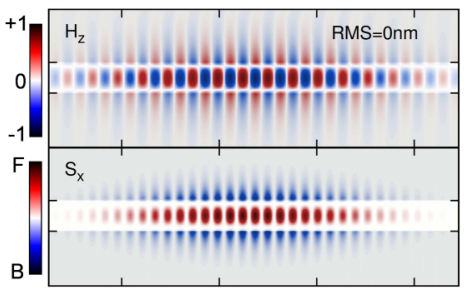

(b)

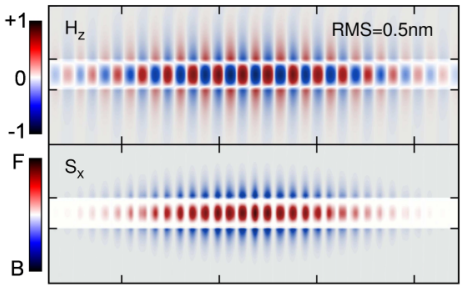

(c)

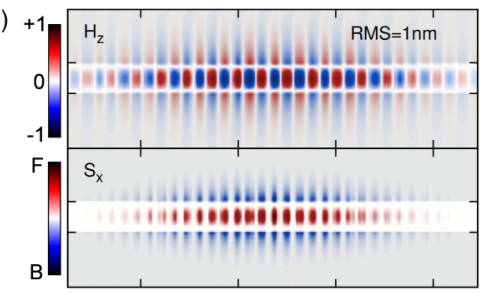

(d)

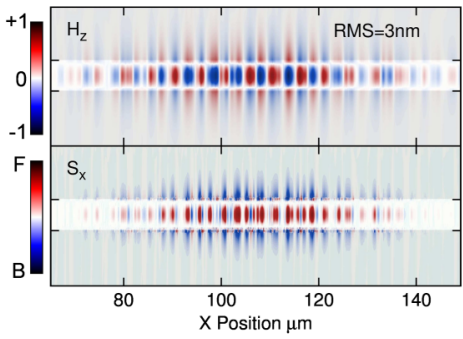

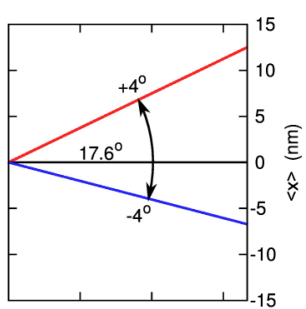
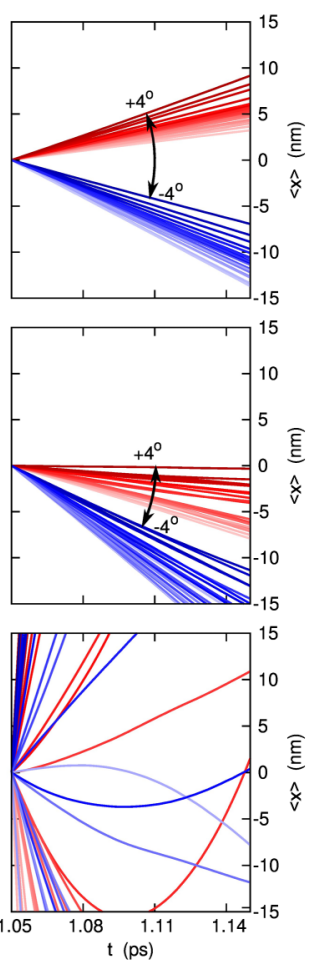

FIG. 4 (color online). (a)-(d) (left column) Two-dimensional ( $x z$-plane) spatial distribution of the magnetic field component $\left(H_{z}\right)$ and Poynting flux $\left(S_{x}\right)$ of the excited light pulse for different rms amplitudes of surface roughness $(\mathrm{rms}=0-3 \mathrm{~nm})$. In the right column, shown are the temporal variations of the mean energy position $\langle x\rangle$ of the excited pulse, inside the waveguide, for illumination angles of the exciting light beam between $-4^{\circ}$ and $+4^{\circ}$, compared with the "critical" angle (for which the pulse stops in the smooth waveguide). For rms roughness up to $1 \mathrm{~nm}$, the pulse travels with a constant velocity (all $\langle x\rangle$ vs $t$ curves are straight lines) and a clear angular dependency, thus retaining a critical angle (shifted with respect to the smooth case) for which the pulse can be completely stopped. By contrast, larger degrees of surface roughness $[\mathrm{rms}=3 \mathrm{~nm}$, (d)] completely change the nature of the pulse through backreflections, destroying the zero group velocity point.

emphasize that the attained lifetimes at the stopping point ( $e^{-1}$ lifetime $28.4 \mathrm{fs}$, and $131 \mathrm{fs}$ for full absorption) are typical nanoplasmonic ones [31], suggesting that approaching the stopping point does not impractically increase losses. For the sake of completeness, we have also calculated that $99 \%$ of the total losses are Ohmic losses, and only $1 \%$ is due to radiation losses. However, we should remark that the efficiency of the stopped-light pulses

generation is currently low, as most of the incident light $(\approx 99 \%)$ is either reflected or absorbed. The amplitude of the stopped pulses can be increased either by the use of gain media $[6,7]$ or by switching off the pump faster.

Finally, we examine the effect of realistic levels of surface roughness [33,34] on the light-stopping ability of the plasmonic heterostructure. To this end, we introduce random variations in the permittivities of the FDTD grid cells surrounding both the Si/ITO and the air/ITO interfaces. The magnitude of the roughness' peak-to-peak variation follows a normal distribution. We investigated the realistic cases of surface roughness rms values of $0.5,1$, and $3 \mathrm{~nm}$ (peak-to-peak values, $\Delta / 2$, are four times those values) $[33,34]$, and in each case we recorded with high precision the temporal evolution of the center of energy $\langle x\rangle$ of an incoupled pulse for various angles of incidence (Fig. 4, right column). From the right panel of Fig. 4(a), we see that in the absence of roughness the incoupled pulse remains exactly immobilized when it is injected inside the heterostructure with an angle $\theta_{c} \sim 17.6^{\circ}$. For angles $\pm 4^{\circ}$ around that critical angle, the pulse moves forwards or backwards, respectively. When roughness of rms value $0.5 \mathrm{~nm}$ is introduced [Fig. 4(b)] we find that, importantly, the pulse retains its shape [i.e., its envelope is not broken into random pulsations (see left panel)] and for a range of angles around $\pm 4^{\circ}$ the pulse moves coherently and with a constant velocity either forwards or backwards. Thus, there still is a critical angle, within the cone in the right-hand panel of Fig. 4(b), for which the pulse can be completely stopped. When the roughness rms value increases to $1 \mathrm{~nm}$ [Fig. 4(c)], we find that for all incidence angles around $\pm 4^{\circ}$ the pulse moves backwards with a constant velocity [the curves in the right-hand panel of Fig. 4(c) remain straight lines], as the angles cone has now moved downwards, below $\langle x\rangle=0$; hence, in this case, too, a larger, positive angle of incidence is required for the injected pulse to be stopped. By contrast, when the rms roughness increases further to $3 \mathrm{~nm}$ [Fig. 4(d)], the pulse envelope is broken due to scattering of the energy at the rough interfaces (visible in the $x$ component of the Poynting vector; left panel) and the center of energy no longer moves with a constant velocity (right panel) but is only dependent on the local roughness topology.

The simplicity of the final design, together with the gigantic enhancement of the group refractive index $\left(\sim 15 \times 10^{6}\right)$ and the minor increase of optical losses (by a factor less than 1.4), make the structure appealing for a host of nanoplasmonic applications. Particularly, the enhancement of the density of states in the region around a flat band is expected to enable ultra-high-efficiency photovoltaics [35], as well as to give rise to strong interactions between quantum emitters and the plasmonic nanostructure $[6,7]$ - the cornerstone of strong optical nonlinearity. We anticipate that this might be extendable potentially even to the single-photon level [10], allowing 
for nonlinear quantum-optical logic operations on truly nanoscopic scales.

K. L. T., T.W.P., and J.M.H. contributed equally to this Letter. We acknowledge useful discussions with John Pendry. This work was supported by The Royal Academy of Engineering, the U.K. Engineering and Physical Sciences Research Council, and the Leverhulme Trust.

*Present address: NSF Nanoscale Science and Engineering Center, University of California, 3112 Etcheverry Hall, Berkeley, California 94720, USA.

k.tsakmakidis@berkeley.edu †.hess@imperial.ac.uk

[1] R. W. Boyd and D. J. Gauthier, Prog. Opt. 43, 497 (2002).

[2] L. Brillouin, Wave Propagation and Group Velocity (Academic, New York, 1960).

[3] P. W. Milonni, Fast Light, Slow Light, and Left-Handed Light (Institute of Physics, Bristol, 2005).

[4] K. L. Tsakmakidis and O. Hess, in Proceedings of the 14th International Conference on Transparent Optical Networks (ICTON), Coventry, 2012 (IEEE, New York, 2012).

[5] K. L. Tsakmakidis, J. M. Hamm, T. W. Pickering, and O. Hess, in Proceedings of Frontiers in Optics 2012/Laser Science XXVIII, Rochester, 2012 (Optical Society of America, Washington, DC, 2012).

[6] O. Hess, J. B. Pendry, S. A. Maier, R. F. Oulton, J. M. Hamm, and K. L. Tsakmakidis, Nat. Mater. 11, 573 (2012).

[7] O. Hess and K. L. Tsakmakidis, Science 339, 654 (2013).

[8] A. Aubry, D. Yuan, A. I. Fernández-Domínguez, Y. Sonnefraud, S. A. Maier, and J. B. Pendry, Nano Lett. 10, 2574 (2010).

[9] M. S. Jang and H. Atwater, Phys. Rev. Lett. 107, 207401 (2011).

[10] Z. Jacob and V. M. Shalaev, Science 334, 463 (2011).

[11] N. Liu, M. Hentschel, Th. Weiss, A. P. Alivisatos, and H. Giessen, Science 332, 1407 (2011).

[12] L. V. Hau, S. E. Harris, Z. Dutton, and C. H. Behroozi, Nature (London) 397, 594 (1999).

[13] S. Zhang, D. A. Genov, Y. Wang, M. Liu, and X. Zhang, Phys. Rev. Lett. 101, 047401 (2008).
[14] N. Papasimakis, V. A. Fedotov, N. I. Zheludev, and S. L. Prosvirnin, Phys. Rev. Lett. 101, 253903 (2008).

[15] M. D. Lukin and A. Imamoğlu, Nature (London) 413, 273 (2001).

[16] C. Liu, Z. Dutton, C. H. Behroozi, and L. V. Hau, Nature (London) 409, 490 (2001).

[17] T. Baba, Nat. Photonics 2, 465 (2008).

[18] S. Mookherjea and A. Oh, Opt. Lett. 32, 289 (2007).

[19] R. J. P. Engelen, D. Mori, T. Baba, and L. Kuipers, Phys. Rev. Lett. 101, 103901 (2008).

[20] Metamaterials: Theory, Design, and Applications, edited by T. J. Cui, D. R. Smith, and R. Liu (Springer, New York, 2009).

[21] See Supplemental Material at http://link.aps.org/ supplemental/10.1103/PhysRevLett.112.167401, which includes Refs. [20-22].

[22] M. S. Kwon and S. Y. Shin, Opt. Commun. 233, 119 (2004).

[23] M. L. Boas, Mathematical Methods in the Physical Sciences (John Wiley \& Sons, New York, 2006), 3rd ed.

[24] A. Bakhtazad, H. Abiri, and R. Ghayour, J. Lightwave Technol. 15, 383 (1997).

[25] E. I. Kirby, J. M. Hamm, T. W. Pickering, K. L. Tsakmakidis, and O. Hess, Phys. Rev. B 84, 041103(R) (2011).

[26] A. Archambault, M. Besbes, and J.-J. Greffet, Phys. Rev. Lett. 109, 097405 (2012).

[27] A. Boltasseva and H. A. Atwater, Science 331, 290 (2011).

[28] K. L. Tsakmakidis, A. D. Boardman, and O. Hess, Nature (London) 450, 397 (2007).

[29] Q. Gan, Z. Fu, Y. J. Ding, and F. J. Bartoli, Phys. Rev. Lett. 100, 256803 (2008).

[30] J. B. Pendry, Science 306, 1353 (2004).

[31] S. A. Maier, Plasmonics: Fundamentals and Applications (Springer, New York, 2007).

[32] R. Ruppin, Phys. Lett. A 299, 309 (2002).

[33] K.-B. Kim, Y.-H. Tak, Y.-S. Han, K.-H. Baik, M.-H. Yoon, and M.-H. Lee, Jpn. J. Appl. Phys. 42, L438 (2003).

[34] P. Chaturvedi, W. Wu, V. J. Logeeswaran, Z. Yu, M. S. Islam, S. Y. Wang, R. S. Williams, and N. X. Fang, Appl. Phys. Lett. 96, 043102 (2010).

[35] D. M. Callahan, J. N. Munday, and H. A. Atwater, Nano Lett. 12, 214 (2012). 